\title{
An Optimal Control Approach to the Unit Commitment Problem
}

\author{
F. A. C. C. Fontes* and D. B. M. M. Fontes** and L. A. Roque***
}

\begin{abstract}
The Unit Commitment (UC) problem is a wellknown combinatorial optimization problem arising in operations planning of power systems. It is typically formulated as nonlinear mixed-integer programming problem and has been solved in the literature by a huge variety of optimizations methods, ranging from exact methods (such as dynamic programming, branch-and-bound) to heuristic methods (genetic algorithms, simulated annealing, particle swarm). Here, we start by formulating the UC problem as a mixed-integer optimal control problem, with both binary-valued control variables and real-valued control variables. Then, we use a variable time transformation method to convert the problem into an optimal control problem with only real-valued controls. Finally, this problem is transcribed into a finite-dimensional nonlinear programming problem and solved using an off-the-shelf optimization solver.
\end{abstract}

Index Terms - optimal control; calculus of variations; maximum principle; normality; optimality conditions.

\section{INTRODUCTION}

In this work, we address the Unit Commitment Problem using Optimal Control methodologies. Despite being an highly researched problem with dynamical and multi-period characteristics, it appears that it has not been addressed by optimal control methods before.

The Unit Commitment (UC) problem plays a key role in power system operations, not only because its optimal scheduling might provide huge gains, but also because it maintains system reliability by keeping a proper spinning reserve. The thermal UC problem can be divided into two subproblems: the mixed-integer nonlinear programming problem of determining the on/off state of the generation units for each time period over a scheduling horizon and the nonlinear programming problem of dispatching the load among on-line units. The UC objective is to minimize the total operating cost of the generating units during the scheduling horizon while satisfying a large set of system and operational constraints. Due to its combinatorial nature, multi-period characteristics, and nonlinearities, this problem is highly computational demanding and, thus, it is a hard optimization task solving the UC problem, for real sized systems. The UC problem has been extensively studied in the

F. A. C. C. Fontes is with ISR-Porto and Faculdade de Engenharia, Universidade do Porto, 4200-465 Porto, Portugal. fafle fe.up.pt

D. B. M. M. Fontes is with LIAAD-INESC-TEC L.A., Faculdade de Economia, Universidade do Porto, 4200-464 Porto, Portugal. fontes a fep.up. pt

L. A. C. Roque*** is with Instituto Superior de Engenharia do Porto, 4200-072 Porto, Portugal. lareisep.ipp.pt

The support of FCT/FEDER Project PTDC/EEA-CRO/116014/2009 "Optimal Control in Constrained and Hybrid Nonlinear Systems" and Marie Curie FP7-ITN-264735-SADCO Project "Sensitivity Analysis for Deterministic Controller Design" is acknowledged literature. Several numerical optimization techniques, based both on exact or on approximate algorithms are reported.

Several heuristic approaches based on exact methods have been used such as dynamic programming, mixed-integer programming, benders decomposition, lagrangian relaxation and branch and bound methods, see e.g. [17], [9], [30], [4]. The main drawbacks of these traditional techniques are the high computational time required for large complexity and dimensionality problems. Dynamic programming [17], [24] is a powerful and flexible methodology, owever its suffers from the dimensionality problem, not only in computational time, but also in storage requirements. Recently a stochastic dynamic programming approach to schedule power plants was proposed [26]. In [4] a solution using lagrangian relaxation is proprosed. However, the problem becomes too complex as the number of units increases and there are some difficulties in obtaining feasible solutions. Takriti [30] addresses the unit commitment problem by using mixedinteger programming which is a very hard task when the number of units increases since it requires large memory and leads to large computational time requirements. The branchand-bound method proposed in [9] uses a linear function to represent the fuel consumption and a time-dependent startup cost, but has a exponential growth in the computational time with problem dimension.

More recently, several metaheuristics methods such as evolutionary algorithms and hybrids of the them have been proposed, see e.g. [32], [11], [28], [7], [3]. These approaches have, in general, better performances than the traditional heuristics. The most commonly used metaheuristic methods are simulated annealing [23], [28], evolutionary programming [15], [25], memetic algorithms (MA)[32], particle swarm optimization [36], tabu search [22], [33], and genetic algorithms [16], [29], [10], [27]. For further discussion an comparison of these methodologies, with special focus on metaheuristic methods see [27].

Although the UC problem is an highly researched problem with dynamical and multi-period characteristics, it appears that it has not been addressed before by optimal control methods, except trying to solve the dynamic programming recursion as mentioned. The nearest problems that we have found in the literature using optimal control methods are for the dispatch problem (see [35] and references therein) which considers that it is already decided which are the units that are on.

So, the formulation of the UC problem as a mixedinteger optimal control problem, given in section II, is novel. However, the main contribution is the variable time transformation method, described in Section III, which converts the 
mixed-integer optimal control problem (OCP) into one with only real-valued controls .

Most literature on optimal control deals with problems with only real-valued controls, both the analytical methods based on variational analysis (see e.g. [34], [8]) and also numerical schemes ([6], [5]). There are, however, some works that are able to address optimal control problems (OCP) with discrete control sets (see e.g. [12], [21]), although dealing directly with the discrete-valued controls is computationally heavy. The transformation of a mixed-integer optimal control problem into a problem with only real-valued controls is not new, nor is new the general idea of a variable time transformation method. See the classical reference [14] and also [31], [19], [20], [2], [18]. See also the recent work [13] for a discussion of several variable time transformation methods.

Although the approach in [13] could, in principle, be used for the UC problem, it would require explicit enumeration of all possible combinations of the control set, which in our case would lead to $2^{N}$ (with $N$ being the number of thermal units) discrete control values and would be impractical. So, we can consider our variable time transformation a suitable modification of [13] when the discrete-valued controls are not scalar, that is valid for the UC problem.

\section{The Unit Commitment Problem}

The schedule of power system involves the solution to the unit commitment problem and the economic dispatch problem (EDP), where the UC problem is an integer programming problem and the EDP is a nonlinear programming problem. Both the UC problem and the EDP are usually referred as the UC problems, which can be modeled as a nonlinear, non-covex and mixed integer combinatorial optimization problem [10]. The thermal unit commitment (UC) has been traditionally solved in centralized power systems to decide when each unit is turned on or turned off along a predefined time horizon. In addition, for each time period one needs to decide on how to dispatch online generators. The objective of the UC problem is the minimization of the total operating costs over the scheduling horizon while satisfying the system demand, spinning reserve requirement and other generation constraints such as the capacity limits, ramp rate limits and minimum up/down times. Therefore, the objective function is expressed by the sum of the fuel and start-up costs. The model has two types of control variables. On the one hand, binary control variables $u_{j}(t)$, which are either set to 1 , meaning that unit $\mathrm{j}$ is committed at time period t; or otherwise are set to zero. On the other hand, real valued variables $\Delta_{j}(t)$, which enable to control the amount of energy produced by unit $\mathrm{j}$ at time period $\mathrm{t}$. Before giving the mathematical formulation let us introduce the parameters and variables notation.

Indexes:

t: Time period index;

j: Generation unit index;
For convenience, let us also define the index sets:

$\mathcal{T}:=\{1, \ldots, T\}$

$\mathcal{J}:=\{1,2, \ldots, N\}$

\section{Decision/Control Variables:}

$\Delta_{j}(t)$ : Amount of thermal generation of unit $j$ to be incremented or decremented comparatively to the production at the previous time period;

$\mathbf{u}_{\mathbf{j}}(\mathbf{t})$ : Status of unit $j$ at time period $t$ ( 1 if the unit is on; 0 otherwise);

\section{State Variables:}

$\mathbf{y}_{\mathbf{j}}(\mathbf{t})$ : Thermal generation of unit $j$ at time period $t$, in $[M W]$;

$\mathbf{T}_{\mathbf{j}}^{\mathbf{o n} / \mathbf{o f f}}(\mathbf{t})$ :Number of time periods for which unit $j$ has been continuously on-line/off-line until time period $t$, in [hours];

$\overline{\mathbf{Y}}_{\mathbf{j}}(\mathbf{t})$ : Maximum allowed generation at time period $t$, considering the ramp limit of unit $j$, in $[M W]$;

\section{Parameters:}

T: Number of time periods (hours) of the scheduling time horizon;

$\mathbf{N}$ : Number of generation units;

$\mathbf{R}(\mathbf{t})$ : System spinning reserve requirements at time period $t$, in $[M W]$;

$\mathbf{D}(\mathbf{t})$ : Load demand at time period $t$, in $[M W]$;

$\mathbf{Y m i n}_{\mathbf{j}}$ : Minimum generation limit of unit $j$, in $[M W]$;

$Y \max _{\mathbf{j}}$ : Maximum generation limit of unit $j$, in $[M W]$;

$\mathbf{T}_{\mathbf{c}, \mathbf{j}}$ : Cold start time of unit $j$, in [hours];

$\mathbf{T}_{\mathbf{m i n}, \mathbf{j}}^{\mathbf{o n} / \text { off }}$ : Minimum uptime/downtime of unit $j$, in [hours];

$\mathbf{S}_{\mathbf{H} / \mathbf{C}, \mathbf{j}}$ : Hot/Cold start-up cost of unit $j$, in $[\$] ;$

$\Delta_{j}^{d n / u p}$ : Maximum allowed output level decrease/increase in consecutive periods for unit $j$, in $[M W]$;

\section{A. Objective Function}

The objective of the UC problem is the minimization of the total costs for the whole planning period, in which the total costs are expressed as the sum of fuel and start-up costs of the generating units. Therefore, the objective function is as follows:

Minimize

$\sum_{t=1}^{T}\left(\sum_{j=1}^{N}\left\{F_{j}\left(y_{j}(t)\right) \cdot u_{j}(t)+S_{j}(t) \cdot\left(1-u_{j}(t-1)\right) \cdot u_{j}(t)\right\}\right)$.

where, the generation costs, i.e. the fuel costs, are conventionally given by a quadratic cost function as follows:

$$
F_{j}\left(y_{j}(t)\right)=a_{j} \cdot\left(y_{j}(t)\right)^{2}+b_{j} \cdot y_{j}(t)+c_{j}
$$

with the cost coefficients $a_{j}, b_{j}, c_{j}$ of unit $j$. The start-up costs, that depend on the number of time periods during which the unit has been off, are given by 
$S_{j}(t)= \begin{cases}S_{H, j}, & \text { if } T_{m i n, j}^{o f f} \leq T_{j}^{o f f}(t) \leq T_{m i n, j}^{o f f}+T_{c, j}, \\ S_{C, j}, & \text { if } T_{j}^{o f f}(t)>T_{m i n, j}^{o f f}+T_{c, j},\end{cases}$

where $S_{H, j}$ is the hot start-up cost and $S_{C, j}$ is the cold start-up cost of unit $j$.

The state dynamics in this model are given by the follows equations:

The thermal production of each unit, at time period $t$, depends of the amount of thermal production in previous time period and is limited by the maximum allowed decrease and increase of the output that can occur during one time period

$$
y_{j}(t)=\left[y_{j}(t-1)+\Delta_{j}(t)\right] \cdot u_{j}(t)
$$

for $t \in \mathcal{T}$ and $j \in \mathcal{J}$, with $\Delta_{j}(t) \in\left[-\Delta_{j}^{d n}, \Delta_{j}^{u p}\right]$. The number of time periods continuously on-line until time period $t$ is given by

$$
T_{j}^{o n}(t)=\left[T_{j}^{o n}(t-1)+1\right] \cdot u_{j}(t),
$$

for $t \in \mathcal{T}$ and $j \in \mathcal{J}$ The number of time periods continuously off-line until time period $t$ is given by

$$
T_{j}^{o f f}(t)=\left[T_{j}^{o f f}(t-1)+1\right] \cdot\left(1-u_{j}(t)\right),
$$

for $t \in \mathcal{T}$ and $j \in \mathcal{J}$.

Due to the mechanical characteristics and thermal stress limitations the output variation levels of each online unit in two consecutive periods are restricted by maximum ramp rate, both up or down,

$$
\bar{Y}_{j}(t)=\left[y_{j}(t-1)+\Delta_{j}^{u p}\right],
$$

for $t \in \mathcal{T}$ and $j \in \mathcal{J}$.

\section{B. Constraints}

The constraints can be classified into two sets: the demand constraints and the operational generator constraints. The first set of constraints is composed by the load requirements and spinning reserve requirements, which can be written as follows:

\section{1) Power Balance Constraints}

The total amount of power generated at each time period must meet the load demand.

$$
\sum_{j=1}^{N} y_{j}(t) \cdot u_{j}(t) \geq D(t), t \in\{1,2, \ldots, T\} .
$$

\section{2) Spinning Reserve Constraints}

The spinning reserve is the total amount of real power generation available from on-line units net of their current production level. The reserve power available is used when a unit fails or an unexpected increase in load occurs.

$$
\sum_{j=1}^{N} \bar{Y}_{j}(t) \cdot u_{j}(t) \geq R(t)+D(t), t \in\{1,2, \ldots, T\} .
$$

The second set of constrains includes unit capacity limits and the minimum number of time periods that the unit must be continuously in each status (on-line or off-line).

\section{3) Unit Capacity Limits}

Each unit has a maximum and minimum output limits.

$$
\begin{aligned}
& Y \min _{j} \cdot u_{j}(t) \leq y_{j}(t) \leq Y \max _{j} \cdot u_{j}(t), \\
& \text { for } t \in\{1,2, \ldots, T\} \text { and } j \in\{1,2, \ldots, N\} .
\end{aligned}
$$

\section{4) Minimum Uptime/Downtime Constraints}

The unit cannot be shut down or started-up instantaneously once it is committed or decommitted. The minimum uptime/downtime constraints indicate that there is a minimum time that each unit must be on-line or off-line, respectively.

$$
\begin{gathered}
\left(T_{j}^{o n}(t-1)-T_{m i n, j}^{o n}\right) \cdot\left(u_{j}(t-1)-u_{j}(t)\right) \geq 0, \\
\quad \text { for } t \in\{1,2, \ldots, T\} \text { and } j \in\{1,2, \ldots, N\} .
\end{gathered}
$$

$$
\begin{aligned}
& \left(T_{j}^{o f f}(t-1)-T_{m i n, j}^{o f f}\right) \cdot\left(u_{j}(t)-u_{j}(t-1)\right) \geq 0, \\
& \quad \text { for } t \in\{1,2, \ldots, T\} \text { and } j \in\{1,2, \ldots, N\} .
\end{aligned}
$$

\section{5) Initial state constraints}

The values of $T_{j}^{o f f}(0)$ and $T_{j}^{o n}(0)$ are given for the initial time. The values of $u_{j}(0)$ are defined accordingly (i.e. set to one if $T_{j}^{o n}(0)$ is positive, set to zero otherwise). The values of $y_{j}(0)$ can be chosen satisfying

$$
Y \min _{j} \cdot u_{j}(0) \leq y_{j}(0) \leq Y \max _{j} \cdot u_{j}(0) .
$$

\section{The Variable Time Transformation Method}

The idea here is to develop a variable time transformation in order to convert the mixed-integer OCP into an OCP with only real-valued controls.

Consider, for each unit $j$, a non-decreasing real-valued function $t \mapsto \tau_{j}(t)$. Consider also a set of values $\bar{\tau}_{1}, \bar{\tau}_{2}, \ldots$ such that when $\tau_{j}(t)=\bar{\tau}_{k}$ for odd $k$ we have a transition from off to on in unit $j$, and when $\tau_{j}(t)=\bar{\tau}_{k}$ for even $k$ we have a transition from on to off. So, we consider that unit $j$ is:

- on if $\tau_{j}(t) \in\left[\bar{\tau}_{1}, \bar{\tau}_{2}\right) \cup\left[\bar{\tau}_{3}, \bar{\tau}_{4}\right) \cup \ldots \cup\left[\bar{\tau}_{2 k-1}, \bar{\tau}_{2 k}\right)$;

- off if $\tau_{j}(t) \in\left[0, \bar{\tau}_{1}\right) \cup\left[\bar{\tau}_{2}, \bar{\tau}_{3}\right) \cup \ldots \cup\left[\bar{\tau}_{2 k}, \bar{\tau}_{2 k+1}\right)$.

An illustrative example is shown in Fig. 1.

It might help to interpret $\tau_{j}$ to be a transformed time scale and that the values $\bar{\tau}_{1}, \bar{\tau}_{2}, \ldots$ are switching "times" in the transformed time scale. We can consider, without loss of generality, that the values $\bar{\tau}_{k}$ are equidistant. Nevertheless, in real time $t$, the distance between two events $\bar{\tau}_{k}$ and $\bar{\tau}_{k+1}$ can be stretched or shrunk to any non-negative value, including zero, depending on the shape of the function $t \mapsto \tau_{j}(t)$.

To simplify exposition, and without loss of generality, let us consider that $\tau_{k}-\tau_{k-1}$ is constant and equal to 1 , for all $k=1,2, \ldots$.

Now, consider that we have the controls

$$
w(t) \in[0,1], \quad t=0,1, \ldots, T-1,
$$

that represent the increment from $\tau(t)$ to $\tau(t+1)$ such that

$$
\tau(t)=\tau_{0}+\sum_{k}^{t-1} w_{(k)}
$$



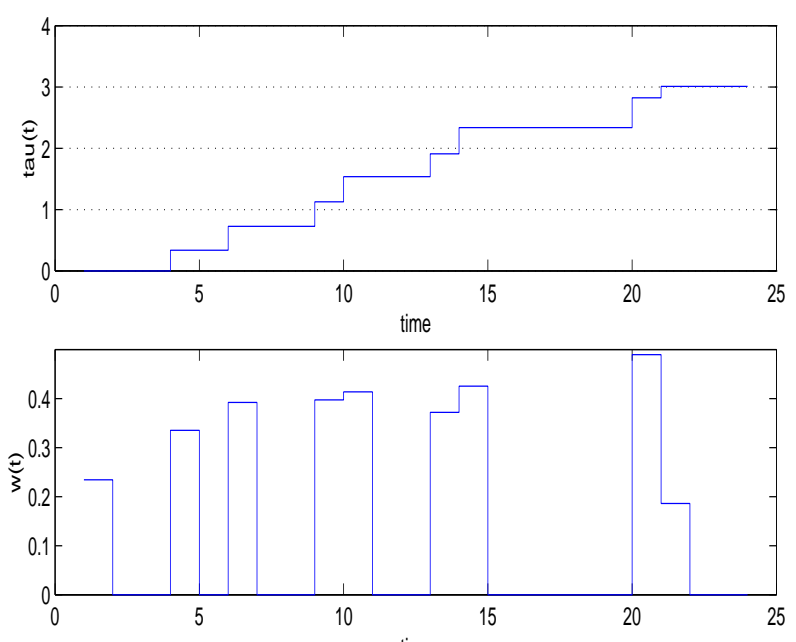

time

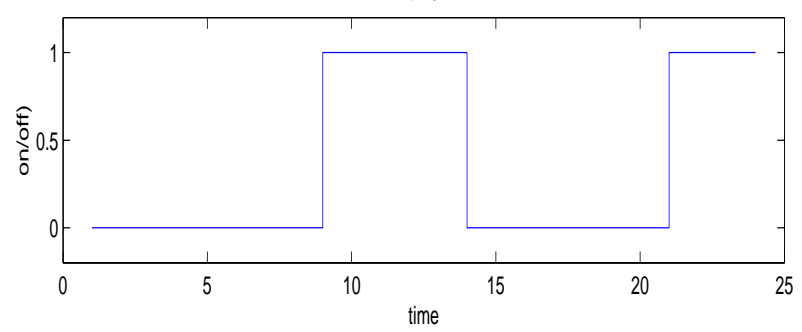

Fig. 1. Example of a function $\tau$, the corresponding on/off status, and the corresponding function $w$

or

$$
w(t)=\tau(t+1)-\tau(t), \quad \text { with } \tau(0)=\tau_{0} .
$$

Possible values of $w$ in our example are shown in Fig. 1.

A. UC problem as an Optimal Control Problem with realvalued controls

We recall the index set $\mathcal{J}$ and redefine $\mathcal{T}$ to be more consistent with usual discrete-time control formulations.

$\mathcal{T}:=\{1, \ldots, T\}$ and $\mathcal{J}:=\{1,2, \ldots, N\}$.

In the same spirit, we redefine the control $\Delta_{j}(t)$ to be the amount of thermal generation incremented or decremented in the next time period (rather than comparatively to the previous period).

We should note that our controls are all real-valued and comprise:

$$
\begin{aligned}
& \Delta_{j}(t) \in\left[-\Delta_{j}^{d n}, \Delta_{j}^{u p}\right], \\
& w_{j}(t) \in[0,1] .
\end{aligned}
$$

Define the sets of time periods:

$I_{j}^{o n}:=\left\{t \in \mathcal{T}: \tau_{j}(t) \in[2 k-1,2 k), k \geq 1\right\}$,

$I_{j}^{o f f}:=\mathcal{T} \backslash I_{j}^{o n}$,

$\begin{aligned} I_{j}^{\text {off }>\text { on }} & :=\left\{t \in \mathcal{T}: \tau_{j}(t) \geq 2 k+1, \tau_{j}(t-1)<2 k+1, k\right. \\ I_{j}^{\text {on }>\text { off }}: & \left.:=\left\{t \in \mathcal{T}: \tau_{j}(t) \geq 2 k, \tau_{j}(t-1)<2 k, k \geq 1\right\}\right\} .\end{aligned}$

Finally, we are able to formulate our OCP:

\section{Minimize}

$$
\sum_{j=1}^{N}\left(\sum_{t \in I_{j}^{o n}} F_{j}\left(y_{j}(t)\right)+\sum_{t \in I_{j}^{o f f}>\text { on }} S_{j}(t)\right)
$$

subject to the dynamic constraints

$$
\begin{aligned}
& \tau_{j}(t+1)=\tau_{j}(t)+w_{j}(t) \quad j \in \mathcal{J}, t \in \mathcal{T} \\
& T_{j}^{o n}(t+1)= \begin{cases}T_{j}^{o n}(t)+1 & j \in \mathcal{J}, t \in I_{j}^{o n} \\
0 & j \in \mathcal{J}, t \in I_{j}^{o f f},\end{cases} \\
& T_{j}^{o f f}(t+1)= \begin{cases}T_{j}^{o f f}(t)+1 & j \in \mathcal{J}, t \in I_{j}^{o f f} \\
0 & j \in \mathcal{J}, t \in I_{j}^{\text {on }},\end{cases} \\
& y_{j}(t+1)= \begin{cases}y_{j}(t)+\Delta_{j}(t) & j \in \mathcal{J}, t \in I_{j}^{\text {on }} \\
0 & j \in \mathcal{J}, t \in I_{j}^{\text {of } f},\end{cases}
\end{aligned}
$$

the initial state constraints

$$
\begin{aligned}
& T_{j}^{o n}(0)=T_{j, 0}^{o n} \quad \text { (given), } \\
& T_{j}^{o f f}(0)=T_{j, 0}^{o f f} \quad \text { (given), } \\
& \tau_{j}(0)= \begin{cases}0 & \text { if } T_{j, 0}^{o n}=0 \\
1 & \text { if } T_{j, 0}^{o n}>0,\end{cases} \\
& y_{j}(0)= \begin{cases}0 & \text { if } T_{j, 0}^{o n}=0 \\
y_{j, 0} \in\left[\min _{j}, Y \max _{j}\right] & \text { if } T_{j, 0}^{o n}>0,\end{cases}
\end{aligned}
$$

the control constraints

$$
\begin{aligned}
& \Delta_{j}(t) \in\left[-\Delta_{j}^{d n}, \Delta_{j}^{u p}\right], \\
& w_{j}(t) \in[0,1],
\end{aligned}
$$

and the pathwise state constraints

$y_{j}(t) \in\left[\min _{j}, Y \max _{j}\right] \quad j \in \mathcal{J}, t \in I_{j}^{\text {on }}$,

$\sum_{j \in \mathcal{J}} y_{j}(t) \geq D(t) \quad t=1,2, \ldots, T$,

$\sum_{j \in \mathcal{J}} \bar{Y}_{j}(t) \geq R(t)+D(t) \quad t=1,2, \ldots, T$

where for each $j \in \mathcal{J}$

$$
\begin{gathered}
\bar{Y}_{j}(t)= \begin{cases}\min \left\{y_{j}(t-1)+\Delta_{j}^{u p}, Y_{\max _{j}}\right\} & t \in I_{j}^{\text {on }} \\
0 & t \in I_{j}^{\text {off }},\end{cases} \\
y_{j}(t) \in\left[Y_{\text {min }_{j}}, \max \left\{Y_{\text {min }_{j}}, \Delta_{j}^{u p}\right\}\right] \quad j \in \mathcal{J}, t \in I_{j}^{\text {off }>\text { on }}
\end{gathered}
$$

$T_{j}^{o n}(t-1) \geq T_{m i n, j}^{o n} \quad j \in \mathcal{J}, t \in I_{j}^{o n>o f f}$

$T_{j}^{o f f}(t-1) \geq T_{m i n, j}^{o f f} \quad j \in \mathcal{J}, t \in I_{j}^{o f f>o n}$.

An example of a possible realization of the functions $\Delta_{j}, w_{j}, \tau_{j}, T_{j}^{o n}, T_{j}^{o f f}, y_{j}$, and $\bar{Y}_{j}$ for a specific unit $j$ is shown in Fig. 2.

\section{Numerical Methodology}

To construct our nonlinear programming problem (NLP), we start by defining the optimization variable $x$ containing 0 both the control and state variables. That is

$$
x=\left[\Delta, w, \tau, T^{o n}, T^{o f f}, y\right]
$$

with dimension $(6 T+1) * N)$. (We could have considered just the controls $\Delta, w$ together with the free initial state $y(0)$. 

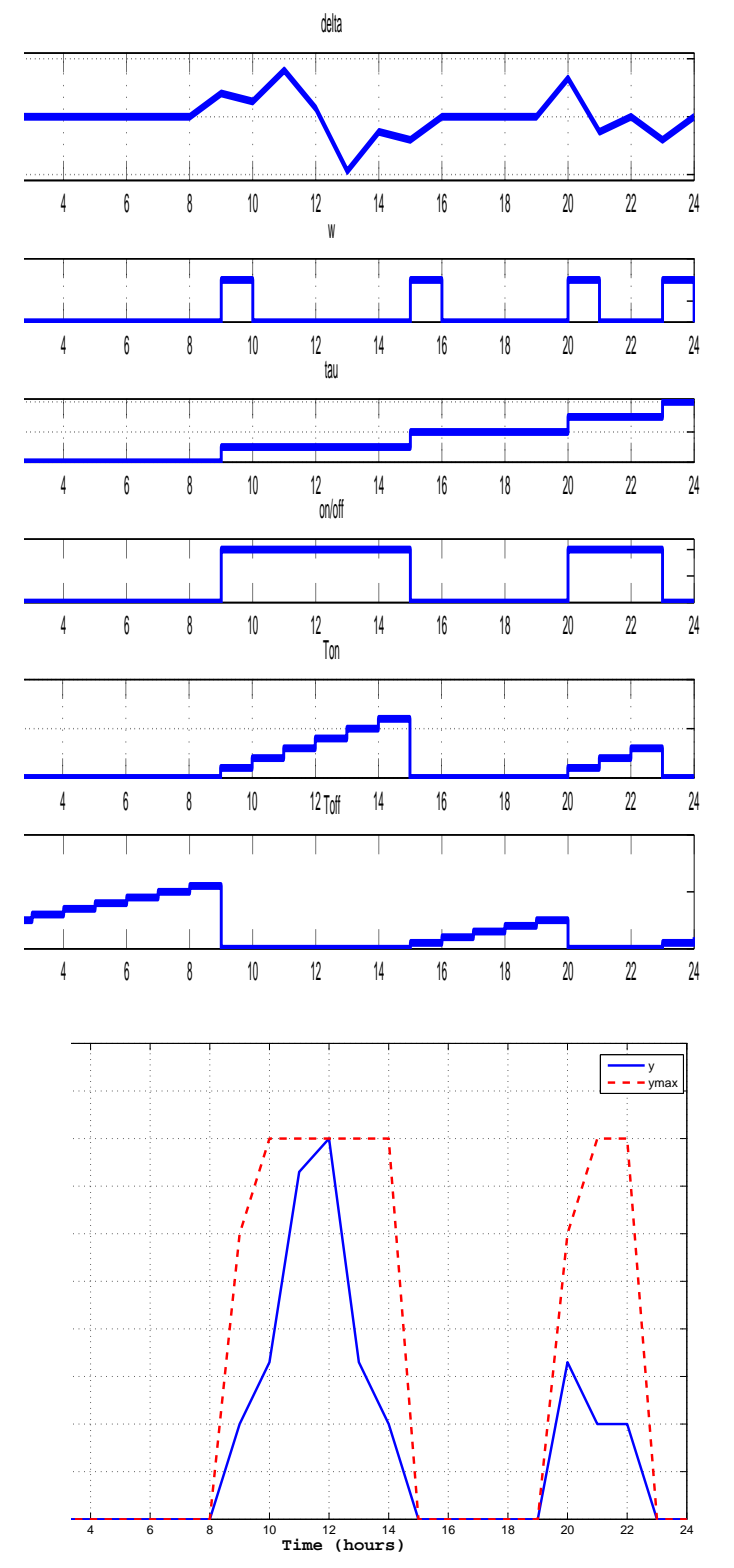

Fig. 2. Example of possible functions $\Delta_{j}, w_{j}, \tau_{j}, T_{j}^{o n}, T_{j}^{o f f}, y_{j}$, and $\bar{Y}_{j}$

An option which, despite having the advantage of a lower dimensional decision variable, is known to frequentely have robustness problems, specially in OCPs with pathwise state constraints such as ours. For further dicussion see e.g. Betts [5].)

To facilitate the optimization algorithm, we separate the constraints that are simple variable bounds, linear equalities, linear inequalities and the remaining:

- upper/lower bounds: equations (22)-(25);

- linear equalities: (15);

- linear inequalities: (26);

- nonlinear equalities: (16)-(18); and

- nonlinear inequalities: (27)-(30).

Note that (19)-(21) are not implemented as constraints since these initial values of state variables are considered as parameters and not variables.

With these considerations the problem is formulated as the following NLP

$$
\begin{aligned}
& \text { Minimize }_{x \in \mathbb{R}^{(6 T+1) \times N}} J(z) \\
& \text { subject to } \\
& \quad L B \leq x \leq U B \\
& A_{\text {eq }} x=b_{\text {eq }} \\
& A_{\text {ineq }} x=b_{\text {ineq }} \\
& g(z)=0 \\
& h(z) \leq 0
\end{aligned}
$$

and solved with an off-the-shelf NLP solver. For the moment we have used Matlab fmincon, but we intend in the future to explore the sparcity of the problem and use another optimizer such as WORHP [1].

The methodology developed was tested in a problem taken from the literature ([16]). The first problem consist of ten units and a time horizon of 24 hours, and the data is given in the Appendix/ Table X (or can be found in e.g. [27].). We have obtained for this problem the cost 565827, which appears (since it is the best value found by several authors) to be an optimal solution to this problem.

\section{CONCLUSIONS}

We have addressed the Unit Commitment problem using optimal control methods, which appears that has not been done previously. In order to solve the mixed-integer optimal control problem (OCP), we have converted it into another OCP with only real-valued controls. This process required a novel variable time transformation that was able to address adequately several discrete-valued control variables arising in the original problem formulation. The transformed real OCP was transcribed into a nonlinear programming problem to be solved by a standard nonlinear optimization solver.

\section{REFERENCES}

[1] WORHP large-scale sparse nonlinear optimization.

[2] Siburian A. Numerical methods for robust, singular and discrete valued optimal control problems. Ph.d. thesis, Curtin University of Technology, Perth, Australia, 2004.

[3] K. Abookazemi, M.W. Mustafa, and H. Ahmad. Structured Genetic Algorithm Technique for Unit Commitment Problem. International Journal of Recent Trends in Engineering, 1(3):135-139, 2009.

[4] A.G. Bakirtzis and C.E. Zoumas. Lambda of Lagrangian Relaxation Solution to Unit Commitment Problem. Proc. Inst. Elect. Eng., Generation, Transmission, Distribution, 147(2):131-136, 2000.

[5] J. Betts. Practical methods for optimal control and estimation using nonlinear programming. SIAM, 2nd edition, 2010.

[6] A. E. Bryson and Y. C. Ho. Applied Optimal Control. John Wiley and Sons, New York, 1975.

[7] Y.M. Chen and W.S. Wang. Fast solution technique for unit commitment by particle swarm optimisation and genetic algorithm. International Journal of Energy Technology and Policy, 5(4):440-456, 2007.

[8] F. H. Clarke. Optimization and Nonsmooth Analysis. WileyInterscience, New York, 1983.

[9] A. I. Cohen and M. Yoshimura. A Branch-and-Bound Algorithm for Unit Commitment. IEEE Transactions on Power Apparatus and Systems, 102(2):444-451, 1983. 


\section{CONFIDENTIAL. Limited circulation. For review only.}

[10] C. Dang and M. Li. A floating-point genetic algorithm for solving the unit commitment problem. European Journal Operational Research, 181(4):1370-1395, 2007.

[11] G. Dudek. Unit commitment by genetic algorithm with specialized search operators. Electric Power Systems Research, 72(3):299-308, 2004.

[12] Khmelnitsky E. A combinatorial, graph-based solution method for a class of continuous-time optimal control problems. Mathematics of Operations Research, 27:312-325, 2002.

[13] Matthias Gerdts. A variable time transformation method for mixedinteger optimal control problems. Optim. Control Appl. Meth., 27:169182,2006

[14] AD Ioffe and Tihomirov VM. Theory of Extremal Problems, volume 6 of Studies in Mathematics and its Applications. North-Holland Publishing Company, Amsterdam, New York, Oxford, 1979.

[15] K.A. Juste, H. Kita, E. Tanaka, and J. Hasegawa. An evolutionary programming solution to the unit commitment problem. IEEE Transactions on Power Systems, 14(4):1452-1459, 1999.

[16] S.A. Kazarlis, A.G. Bakirtzis, and V. Petridis. A Genetic Algorithm Solution to the Unit Commitment Problem. IEEE Transactions on Power Systems, 11:83-92, 1996.

[17] F.N. Lee. Short-term unit commitment-a new method. IEEE Transactions on Power Systems, 3(2):421-428, 1998.

[18] H W J Lee, K L Teo, and X Q Cai. An optimal control approach to nonlinear mixed integer programming problems. Computers and Mathematics with Applications, 36:87-105, 1998.

[19] H W J Lee, K L Teo, V Rehbock, and L S Jennings. Control parameterization enhancing technique for time optimal control problems. Dynamic Systems and Applications, 6:243-262, 1997.

[20] H W J Lee, K L Teo, V Rehbock, and L S Jennings. Control parametrization enhancing technique for optimal discretevalued control problems. Automatica, 35:1401-1407, 1999.

[21] Gerdts M. Solving mixed-integer optimal control problems by branch and bound: a case study from automobile testdriving with gear shift. Optimal Control, Applications and Methods, 26:1-18, 2005.

[22] A.H. Mantawy, Y.L. Abdel-Magid, and S.Z. Selim. Unit commitment by tabu search. IEE Proceedings Generation, Transmition, Distribution, 145(1):56-64, 1998.

[23] A.H. Mantawy, L. Abdel-Magid Youssef, and Z. Selim Shokri. A simulated annealing algorithm for unit commitment. IEEE Transactions on Power Systems, 13(1):197-204, 1998.

[24] N.P. Padhy. Unit Commitment using Hybrid Models: A Comparative Study for Dynamic Programming, Expert Systems, Fuzzy System and Genetic Algorithm. International Journal of Electrical Power \& Energy Systems, 23(8):827-836, 2001

[25] C.C.A. Rajan and M.R. Mohan. An evolutionary programming-based tabu search method for solving the unit commitment problem. IEEE Transactions on Power Systems, 19(1):577-585, 2004.

[26] S. Rebennack, B. Flach, M.V.P. Pereira, and P.M. Pardalos. Stochastic Hydro-Thermal Scheduling under $\mathrm{CO} 2$ Emission Constraints. IEEE Transactions on Power Systems, 27(1):58-68, 2012.

[27] L. Roque, D. B. M. M Fontes, and F. A. C. C. Fontes. A Biased Random Key Genetic Algorithm Approach for Unit Commitment Problem. Lecture Notes in Computer Science, 6630(1):327-339, 2011.

[28] D.N. Simopoulos, S.D. Kavatza, and C.D. Vournas. Unit commitment by an enhanced simulated annealing algorithm. IEEE Transactions on Power Systems, 21(1):68-76, 2006.

[29] K.S. Swarup and S. Yamashiro. Unit Commitment Solution Methodology Using Genetic Algorithm. IEEE Transactions on Power Systems, 17:87-91, 2002.

[30] S. Takriti and J.R. Birge. Using Integer Programming To Refine Lagrangian-Based Unit Commitment Solutions. IEEE Transaction on Power Systems, 15(1):151-156, 2000.

[31] K L Teo, L S Jennings, and V Rehbock. The control parameterization enhancing transform for constrained optimal control problems. Journal of the Australian Mathematics Society, 40:314-335, 1999.

[32] J. Valenzuela and A.E. Smith. A seeded memetic algorithm for large unit commitment problems. Journal of Heuristics, 8(2):173-195, 2002.

[33] T.A.A. Victoire and A.E. Jeyakumar. A tabu search based hybrid optimization approach for a fuzzy modelled unit commitment problem. Electric Power Systems Research, 76:413-425, 2006.

[34] R. Vinter. Optimal control. Birkhauser, Boston, 2000.

[35] X. Xia and A.M. Elaiw. Optimal dynamic economic dispatch of generation: A review. Electric Power Systems Research, 80:975-986, 2010 .
[36] B. Zhao, C.X. Guo, B.R. Bai, and Y.J. Cao. An improved particle swarm optimization algorithm for unit commitment. International Journal of Electrical Power \& Energy Systems, 28(7):482-490, 2006. 